\title{
Ansprache des Vorsitzenden Christian Starck
}

I.

Die mit den Jahreszahlen 1949 und 1999 bezeichnete Zeitspanne von 50 Jahren bedeutet 50 Jahre Grundgesetz und 50 Jahre Vereinigung der Deutschen Staatsrechtslehrer nach ihrer Wiedergründung. Das Grundgesetzjubiläum wurde im Mai begangen, und viele der hier Versammelten haben daran durch Vorträge und Aufsätze und in Kolloquien aktiv teilgenommen. Es ist von großer symbolischer Bedeutung, daß unsere Vereinigung alsbald nach dem Inkrafttreten des Grundgesetzes im Oktober 1949 wieder gegründet worden ist. ${ }^{1}$

Seit 50 Jahren werden Jahr für Jahr auf unseren Tagungen Probleme der Auslegung des Grundgesetzes erörtert. ${ }^{2}$ Diese kontinuierliche Beschäftigung mit dem Grundgesetz schloß es nach Meinung des Vorstandes aus, auf der diesjährigen Tagung ein besonderes Grundgesetzfest zu veranstalten und die Referenten um allgemeine Würdigungen des Grundgesetzes nach 50 Jahren zu bitten. Vielmehr fahren wir in unserer normalen Arbeit fort, indem wir aktuelle verfassungs- und verwaltungsrechtliche Themen debattieren. Und unser besonderer Beitrag zum Grundgesetzjubiläum besteht einfach darin, daß dies in einer ununterbrochenen Kontinuität nun im 50. Jahr ${ }^{3}$ geschieht. Der 50. Jahrestag der Wiedergründung unserer Vereinigung ist aber gewiß Anlaß, sich kurz zu vergewissern, was getan und was versäumt worden ist und wie es in Zukunft weitergehen soll.

${ }_{1}^{1}$ Zur Frage, ob die Vereinigung nach 1933 rechtswirksam aufgelöst wurde oder nur inaktiv war, vgl. Konrad Hesse, AöR 99 (1974), S. 312 f.; Hans-Peter Ipsen, VVDStRL 52 (1993), S. 7 ff.; Micbael Siolleis, Geschichte des öffentlichen Rechts in Deutschland, Bd. 3, 1999, S. 311 f.; ders., Die Vereinigung der Deutschen Staatsrechtslehrer. Bemerkungen zu ihrer Geschichte, in: KritV 80 (1997), S. 339, $343 \mathrm{ff}$.

${ }^{2}$ Vgl. die zusammenfassenden Darstellungen von Hans-Peter Ipsen, AöR 97 (1972), S. 375 - 417; AöR 109 (1984), S. 555-593; AöR 117 (1992), S. 595-643. Nachdruck separat: Hans-Peter Ipsen, Staatsrechtslehrer unter dem Grundgesetz, 1993.

${ }^{3}$ Wegen der Sondertagung 1990 haben seit 1949 bereits 51 Tagungen stattgefunden, und 1999 findet die 52. Tagung statt. 
1972 anläßlich der 50. Wiederkehr des Jahrestages der ursprünglichen Gründung der Vereinigung im Jahr 1922 ist daran erinnert worden, ${ }^{4}$ daß Heinrich Triepel, der Initiator der Vereinigung, ihr eine integrierende Aufgabe zugeschrieben hat. ${ }^{5}$ Sie biete die institutionelle Grundlage ${ }_{n}$ sachlicher und letztlich sittlicher Gemeinschaft ${ }^{*}{ }^{6}$ Konrad Hesse hat das, was gemeint ist, in den folgenden Worten beschrieben ${ }^{7}$ : Jeder könnte ohne solche Verbindung in der Mehrzahl seiner Kollegen nur abstrakte Vertreter von wissenschaftlichen Lehren sehen, die er billigt oder die er befehdet: wenn er die Menschen kennt, die hinter den Lehren stehen, wird er auch die wissenschaftlichen Gegenpositionen besser verstehen und darum vorsichtiger, wahrscheinlich auch gerechter beurteilen - eine versachlichende Wirkung dieser Verbindung." Die institutionelle Leitidee unserer Vereinigung besteht also in wissenschaftlicher Diskussion und persönlicher Verbindung unter den Mitgliedern. ${ }^{8}$

Ich habe mir von Zeit zu Zeit die Frage gestellt, was mir die Vereinigung bedeutet, der ich nun seit 30 Jahren angehöre. Es ist die gemeinsame Beschäftigung mit Fragen des öffentlichen Rechts in einer nicht spezialistischen Weise vor breitem Horizont, dabei Festigung und Infragestellung eigener Überzeugungen, Eröffnung neuer Perspektiven oder einfach Lernen. Aufs engste hängt damit zusammen die persönliche Verbindung. Die Aussprachen finden zumeist in einer offenen wissenschaftlichen Atmosphäre statt. Ich empfinde mich nicht nur während der Jahrestagungen, sondern auch in der Zeit dazwischen in einer wissenschaftlichen Gemeinschaft stehend, zugleich herausgefordert und geborgen. Was auf den Jahrestagungen zur Sprache kommt und näher erörtert wird, ist gemeinsames Wissen in seiner Beständigkeit ebenso wie in seiner Fragwürdigkeit.

II.

Gestatten Sie mir zunächst einen Blick zurück auf das Jahr 1949. Vor nunmehr fast genau 50 Jahren, am 21. Oktober 1949, eröffnete der Alters-

\footnotetext{
${ }^{4}$ Konrad Hesse, Zum 50. Jahrestag der Gründung der Vereinigung der Deutschen Staatsrechtslehrer, AöR 97 (1972), S. 345.

${ }^{5}$ Heinrich Triepel, Die Vereinigung der deutschen Staatsrechtslehrer, AöR 43 (1922), S. 349.

6 So Rudolf Smend, Heinrich Triepel, in: ders., Staatsrechtliche Abhandlungen, 2. Aufl. 1968, S. 593, 606.

${ }^{7}$ Hesse (Anm. 4), S. 348.

${ }^{8}$ So faßte Ulrich Scbeuner, VVDStRL 31 (1973), S. 7, den Triepelschen Gründungszweck zusammen.
} 
präsident Richard Thoma in Heidelberg die Versammlung von Staatsrechtslehrern,9 über deren Einberufung ich gestern beim Empfang des Rektors gesprochen habe. Thoma erwähnte kurz die "Jahre der Verwirrung, der Gewaltherrschaft, des verheerendsten Krieges, der unsagbaren Mord- und Greueltaten“. Als Aufgabe der wiedergegründeten Vereinigung bezeichnete er den "Dienst an der richtigen Deutung und wohlerwogenen Fortbildung des Staatsrechts eines demokratischen Rechtsstaates". Als der Rechtsstaat unterwühlt und erstickt gewesen sei, habe sich unsere Vereinigung nicht gleichgeschaltet, sondern ihre Tätigkeit eingestellt; erhobenen Hauptes könne sie jetzt wieder hervortreten.

Die Vereinigung als Institution ist in der Tat unbelastet durch die "Jahre der Verwirrung ${ }^{4}$. Da die Reihe unserer Veröffentlichungen 1932 mit der Dokumentation der Hallenser Tagung abbricht, sind uns Bände z.B. über den Führerbegriff im Staatsrecht, über die Aufgaben der Verwaltung in der völkischen Lebensordnung, über die Verzichtbarkeit von Gewaltenteilung und Grundrechten im Volksstaat usf. erspart geblieben. $\mathrm{Daß}$ sich freilich die Neugründung der Vereinigung nicht auf die institutionelle Seite beschränken ließ, sondern die Lehren und Haltungen ihrer Mitglieder, der Staatsrechtslehrer, in Betracht zu ziehen gewesen wären, ist durchaus gesehen worden. So hat Ernst Friesenbahn 1950 in seiner Bonner Rektoratsrede bemängelt, daß anläßlich der Heidelberger Tagung „die wenig ruhmvolle Haltung der deutschen Staatsrechtswissenschaft ... mit Stillschweigen übergangen" wurde; er sprach von einem gespenstischen Vorgang. ${ }^{10}$

In dem großen Abstand von 50 Jahren vermag man dem verschämten Neuanfang, der so viel verschwieg und ganz auf das Institutionelle abstellte, vielleicht doch einen Sinn abzugewinnen. Es waren vor allem Erich Kaufmann und Walter Jellinek, zwei durch das NS-Regime Geschädigte, die die Neugründung betrieben, in Korrespondenz mit anderen entschieden, welche ehemaligen Mitglieder nicht eingeladen wurden, ${ }^{11}$ und die Heidelberger Tagung organisiert haben. ${ }^{12}$ Der Wunsch nach Wiederaufnahme der Kontakte und wissenschaftlichem Austausch unter den Staatsrechtslehrern führte zu großer Resonanz unter mehr oder weniger Belasteten, den Verfolgten, den zurückgekehrten Emigranten und der Gruppe derjenigen, die die NS-Zeit in Deutschland überstanden hatten,

\footnotetext{
9 VVDStRL 8 (1950), S. 1 f.

${ }^{10}$ Ermst Friesenbahn, Staatsrechtslehrer und Verfassung (Bonner Akademische Reden 4), 1951, S. 7.

${ }^{11}$ Es handelte sich um Reinhard Höhn, Emst Rudolf Huber (der 1956 aufgenommen wurde), Otto Koellreutter und Carl Scbmitt.

12 Vgl. auch zum folgenden Stolleis, KritV 80 (1997), S. 339, 346 f.
} 
ohne sich zu diskreditieren. Allen kam der Umstand zugute, daß die Vereinigung als Institution einen guten Neuanfang ermöglichte, der nicht mit der Vergangenheit belastet war. Man richtete die Augen in die $\mathrm{Zu}-$ kunft. Auch später fand im Rahmen der Vereinigung keine Aussprache über die Rolle der Staatsrechtslehrer während der nationalsozialistischen Diktatur statt. Es wurde Rücksicht auf die Betroffenen genommen, die in ihren wissenschaftlichen Arbeiten das neue Verfassungssystem anerkannten und z. T. mit bedeutsamen Beiträgen daran mitwirkten, es zu festigen.

Inzwischen gibt es zahlreiche Darstellungen des Staatsrechts der Weimarer Zeit, insbesondere unter dem Gesichtspunkt des Methodenstreits, und der nationalsozialistischen Zeit. ${ }^{13}$ Beide Perioden gehören zur Geschichte. Wir sind keine rechtshistorische Gesellschaft, gewiß. Aber mit der neuesten Geschichte des eigenen Faches müssen wir uns beschäftigen. Vorträge und Aussprachen im Kreis unserer Vereinigung bringen die Dinge anders zur Sprache als Aufsätze und Bücher. Deshalb sollte nach Ansicht des gegenwärtigen Vorstandes auf einer der nächsten Tagungen die Rolle der Staatsrechtswissenschaft während des nationalsozialistischen Regimes und ihre Vorgeschichte seit dem Ende des Ersten Weltkrieges ${ }^{14}$ in Vorträgen behandelt und in einer Aussprache weiter erörtert werden. $Z \mathbf{u}$ fragen wäre nach den „Voraussetzungen und Rahmenbedingungen (, die) es ermöglicht haben, daß das geistige und politische Verhängnis des Nationalsozialismus die rechtsethischen Überlieferungen und die rechtsstaatlichen Grundsätze der vorausgegangenen Epochen aus den Angeln gehoben hat ${ }^{\alpha}{ }^{\alpha}$ Dabei braucht es nicht um das persönliche Versagen der nicht mehr Lebenden zu gehen. Notwendig ist eine sachliche Bestandsaufnahme des eigenen Faches in einem schwierigen Umfeld. Diese Bestandsaufnahme muß in einem Abstand von 60 Jahren möglich sein.

III.

Werfen wir noch einen Blick in die Zukunft unserer Vereinigung. Die wissenschaftliche Durchdringung des Verfassungs- und Verwaltungs-

${ }^{13}$ Vgl. Kurt Sontbeimer, Antidemokratisches Denken in der Weimarer Republik, 1962, 4. Aufl. 1994; zuletzt Michael Stolleis, Geschichte des öffentlichen Rechts in Deutschland, Bd. 3, 1999; aus der weiteren Literatur: Emst-Wolfgang Böckenförde (Hrsg.), Staatsrecht und Staatsrechtslehrer im Dritten Reich, 1985.

${ }^{14}$ In Stichworten: Versailles, Wirtschaftskrise, antidemokratische Strömungen, autoritäre und faschistische Tendenzen in anderen europäischen Staaten, Nationalismus und Verdrängen der gemeinsamen europäischen Rechtsgrundsätze, sozialdarwinistische und antihumanistische Strömungen in der Philosophie.

${ }^{15}$ Rüdiger Breuer, Staatsrecht und Gerechtigkeit, in: FS für Redeker, 1993, S. 11, 16. 
rechts unter Beachtung der sich wandelnden Realien bleibt unsere Aufgabe. Dabei müssen wir klar und ohne Schnörkel sprechen. Denn das Recht, das wir vermitteln, ist Sprache. Es muß verständlich sein für die Studenten, die wir lehren, für die Bevölkerung, auf die die Staatsgewalt zurückgeführt wird, und für Ausländer, die unsere Sprache lernen, um unser Recht zu verstehen.

Die Bedeutung des Bundesverfassungsgerichts für die Verfassungsinterpretation und die des Bundesverwaltungsgerichts für die Verwaltungsrechtsdogmatik werden fortbestehen, und zwar als ständige Anregung und Herausforderung der Staatsrechtslehre. Funktionierende Gerichtsbarkeit sichert die Normativität des Rechts - das gilt auch im Hinblick auf den Europäischen Gerichtshof - und verleiht der praktischen Handhabung des Rechts einen Rationalitätsschub, der von der Staatsrechtslehre aufgenommen und verstärkt wird. Die Notwendigkeit, Gerichtsentscheidungen zu begründen, ist oft Anlaß, rechtswissenschaftliche Überlegungen zu verarbeiten. Die Gerichte sind auf solche Arbeiten geradezu angewiesen. So sind wissenschaftliche Vorarbeit und kritische Nacharbeit wesentliche Beiträge der Staatsrechtslehre für eine gedeihliche Verfassungsgerichtsbarkeit. Je mehr verfassungsgerichtliche Entscheidungen - jetzt auch verstärkt von den Landesverfassungsgerichten erlassen werden, um so größer wird das Bedürfnis, deren dogmatische Kongruenz zu würdigen und zu untersuchen, wie weit das Verfassungsrecht reicht und wo die Gestaltungsfreiheit und damit die politische Verantwortung des Gesetzgebers und der Regierung beginnt. Entsprechendes gilt für die nationale und europäische Verwaltungsgerichtsbarkeit.

Die ständige fruchtbare Mitarbeit der österreichischen, schweizerischen, griechischen und französischen Mitglieder in der Vereinigung hat uns bei unserer Arbeit immer den Zugang zur Rechtsvergleichung offen gehalten. Damit blieben die die nationalen Rechtsordnungen überschreitenden allgemeinen Rechtsgrundsätze im Blick. Angesichts der zunehmenden internationalen Zusammenarbeit, einer immer dichter werdenden Gemeinschaftsrechtsordnung sowie der Europäisierung, ja Globalisierung der Probleme in Wirtschaft, Umwelt, Wissenschaft und militärischer Verteidigung ist die Staatsrechtslehre heute mehr denn je dazu aufgerufen, mit den Mitteln der Rechtsvergleichung die juristischen Problemlösungen anderer Rechtsordnungen im Vergleich der verschiedenen normativen und sozialen Ambiance zu studieren und in die Diskussion über die Gesetzgebung und die Verfassungsauslegung einzubringen. ${ }^{16}$ Dabei darf freilich über die Positivität des deutschen Verfassungsrechts nicht hinweggesehen werden.

16 Christian Starck, Rechtsvergleichung im öffentlichen Recht, JZ 1997, S. 1021, 1023 ff. 
Solche Blicke auf allgemeine Rechtsgrundsätze und fremdes Recht bewahren uns davor, die begriffliche Verfeinerung des deutschen Verfassungsrechts, insbesondere die Grundrechtsdogmatik, zu übersteigern, legislatorische Problemlösungen zu erschweren und den verfassungsrechtlichen Rahmen zu eng zu ziehen.

Die Vereinigung hat das Bedürfnis einer verstärkten grenzüberschreitenden Rechtsbetrachtung erkannt, als im letzten Jahr in Potsdam nach längeren Vorberatungen der Arbeitskreis „Europäisches Verfassungsrecht " gegründet und von der Mitgliederversammlung einstimmig bestätigt worden ist. Viele junge Gelehrte, die uns als neue Mitglieder zugewachsen sind, führen in ihrer venia legendi den ausdrücklichen Ausweis der Rechtsvergleichung; sie haben die neuen Aufgaben schon in Angriff genommen!

Eine gute Zukunft vor Augen können wir uns nun unserer Arbeit widmen, indem wir über „Arbeitsmarkt und staatliche Lenkung " sprechen, und zwar in der eben geschilderten Weise, daß insbesondere der 3 . Berichterstatter, Herr von Danwitz, den Blick über die deutschen Grenzen richtet. 